\title{
Predictive precoding based on the Grassmannian manifold for UAV-enabled cache-assisted B5G communication systems
}

\author{
Wen Zhou ${ }^{1 *} \mathbb{D}$, Xutao Li ${ }^{2}$, Haiqing Wu${ }^{1}$, Yihan Xu${ }^{1}$, Qingfeng Zhou ${ }^{3}$ and Yanyi Rao ${ }^{4 *}$
}

*Correspondence:
wenzhou@ustc.edu;
raoyy@gzhu.edu.cn
${ }^{1}$ Department of Electronic
Engineering, Nanjing Forestry
University, Nanjing, China
${ }^{4}$ School of Computer Science and
Cyber Engineering, GuangZhou
University, GuangZhou, China
Full list of author information is
available at the end of the article

Springer Open

\begin{abstract}
The unmanned aerial vehicle (UAV) can extend the network coverage and improve the system throughput for 5 th generation $(5 \mathrm{G})$ communication systems; hence, it receives a lot of attention recently. This paper considers the problem of channel predictive precoding for UAV-enabled cache-assisted B5G multi-input multi-output (MIMO) systems. A novel channel precoder predictor is proposed, in which the prediction is conducted on a non-linear vector space-Grassmannian manifold. The predictor at the receiver utilizes the current and previous channel matrices to solve the precoder at the next time and then feeds it back to the transmitter for precoding. More specifically, two sub-matrices are extracted from the channel right singular matrices and modeled as two points on the Grassmannian manifold. Then, the geodesic between the two points is conducted. Unlike the conventional method in which the tangent vector at the previous point is parallel transported along the geodesic, we predict the next point by use of the geodesic equation directly. We analyze the computational complexity of the proposed method and demonstrate the superiority of the proposed method by comparing with the conventional one. Besides, we adopt a general Ricean channel model in the UAV MIMO system, where both the Kronecker model and Jake's model are incorporated. The effects of various channel model parameters on the system performance in terms of the chordal error of channel predictor and the optimum step are thoroughly investigated.
\end{abstract}

Keywords: Grassmannia manifold, Prediction, UAV, MIMO

\section{Introduction}

In recent years, there has been a rapid progress in the wireless communication [1-3], and many wireless transmission techniques have been proposed to meet the requirement of ultra-reliable and low-latency [4-7]. The unmanned aerial vehicle (UAV), an aerial platform developed with modern communication technologies, is an unmanned and reusable aircraft powered by electricity or fuel. The UAV has wide applications and can be used in intelligence investigation, disaster relief and rescue, precise target location and tracking, etc. The UAV can increase the coverage area of 5th generation (5G) network and increase

(c) The Author(s). 2020 Open Access This article is licensed under a Creative Commons Attribution 4.0 International License, which permits use, sharing, adaptation, distribution and reproduction in any medium or format, as long as you give appropriate credit to the original author(s) and the source, provide a link to the Creative Commons licence, and indicate if changes were made. The images or other third party material in this article are included in the article's Creative Commons licence, unless indicated otherwise in a credit line to the material. If material is not included in the article's Creative Commons licence and your intended use is not permitted by statutory regulation or exceeds the permitted use, you will need to obtain permission directly from the copyright holder. To view a copy of this licence, visit http://creativecommons.org/licenses/by/4.0/. 
the capacity of 5G systems and user equipments (UEs) as well. Therefore, it has received a lot of attention by the industry and academia.

UAV communications usually adopt the mmWave band, since the multi-input multioutput (MIMO) technology is easy to use in this band [8, 9]. The MIMO system, equipped with multiple antennas at both the transmitter and receiver, makes use of spatial resources adequately and has high data throughput and reliable communication quality. Channel tracking/prediction is always a research hot spot in MIMO systems. Channel state information (CSI) is indispensable for the data decoding at the receiver, which is often obtained by channel estimation. Usually, it is completed with the aide of the pilot signal sent by the transmitter frequently. However, by doing so, quite a part of the channel resources are occupied by the pilot signal, especially for fast fading channels. To reduce the length of the pilot signal on the whole, channel tracking or prediction was proposed by some literature [10]. These methods include the Kalman filter-based method [10], extended Kalman filter-based method [11], sequential Monte Carlo filter-based method [12], and particle filter-based method [13]. Recently, there are some works on the channel tracking in UAV MIMO systems. In [14], a channel tracking method for UAV MIMO communication systems was proposed and investigated, in which the method explores the characteristics of time-varying UAV channels with the beam squint effect. In [15], to improve the quality of the UAV navigation, the authors designed a channel tracking algorithm for its flight control system, where the time-varying spatial channel is characterized by a 3D geometry-based channel model and the algorithm incorporates the outputs of multiple sensors in order to reduce the training overhead and energy consumption.

On the other front, channel predictive information can be feeded back to the transmitter for preprocessing in order to improve the system performance in terms of the capacity or the bit-error-rate (BER) [16]. For instance, in [16], the authors proposed a subspace-based channel tracking scheme for precoded MIMO orthogonal frequency division multiplexing (MIMO-OFDM) systems. The predictive CSI was used for precoding at the transmitter to improve the system throughput. With the development of UAV communications, it can be foreknown that there will be a large amount of data to be transmitted interactively between UAVs and ground base stations/terminals. For instance, with the development of forestry informatization, forestry resource management needs more data support. The UAV is suitable for collecting forestry data and sending them back to the ground control center. Besides, the UAV can be used for data offloading of possibly overloaded cellular base stations in hot spots such as sports venues and cinemas. It can also be used for periodic data distribution/collection in large-scale Internet of Things networks. Large amount of data transmission requires temporary storage space. The caching technique pre-stores the data during the off-peak traffic and improves 5G system performance in many aspects [17-19]. With the aid of cache, it is convenient for the UAV to store data for its own use or forward data to other UAVs or ground terminals [20-22]. Clearly, the feedback information of channel prediction can also be used for UAV MIMO systems to improve the quality of data transmission.

Most previous work above is conducted based on the linear vector space, i.e., Euclidean space, which can be viewed as a linear manifold. Recently, with the rise of information geometry, a few channel tracking methods based on the non-linear manifold were proposed and investigated [23-26]. For instance, in [23], the authors first modeled the 
column spaces spanned by the right singular matrix of the MIMO channel as a point of Grassmannian manifold, and then proposed a method to track the movement of the point as well as an adaptive codebook for precoding. In [26], for the time-varying MIMO system, the authors proposed a predictive quantizer for the eigenvectors of the Gramian matrix that is created from the channel matrix, in which the quantizer operates on the compact Stiefel manifold. In this paper, we mainly predict a sub-matrix of the channel right singular matrix, which is created by selecting a few columns of the channel right singular matrix. The sub-matrix has more rows than columns and can be modeled as a point of Grassmannian manifold. When the channel is time varying, it is reasonable to assume that this sub-matrix moves along the geodesic within a short period of time. Therefore, we intend to use the theory and results of Grassmannian manifold in the channel prediction.

For the prediction methods based on Grassmannian manifold, a key parameter in the design is the choice of the search step. However, most previous work did not investigate the parameter thoroughly. Furthermore, considering the complexity of the practical UAV MIMO channel, this article incorporates both the time correlation and spatial correlation of the channel and studies the effects of various system parameters on the chordal error performance. The contributions of this article are briefly listed as follows:

1 We propose a novel channel predictive precoding method which is based on the geodesic equation on the Grassmannian manifold and analyze its computational complexity as well as the conventional one. Compared with the conventional method, although the complexity of the two methods is the same, the proposed method has better prediction performance in terms of the channel chordal error.

2 We adopt a general Ricean channel model which incorporates the time and spatial correlation by combining the Kronecker model and Jake's model. The effects of various system parameters on the system performance in terms of the chordal error of channel predictor as well as the choice of optimal step are thoroughly investigated.

3 In the Appendix, we prove a corollary which is inferred from the geodesic equation and is helpful to verify the correctness of the geodesic equation from two points on the Grassmannian manifold.

The rest of this paper is organized as follows. The closed-loop UAV-enabled MIMO system model is described in Section 2. The process of channel precoding prediction and the corresponding problem of interest are illustrated in Section 3. The effect of various system parameters on the performance of the prediction algorithm is presented in Section 4, followed by conclusions in Section 5 .

Notations: Boldface lowercase letters denote vectors, and boldface uppercase letters denote matrices. The notation $E($.$) represents the statistical expectation; \mathbb{C}^{m \times n}$ and $\mathbb{U}^{m \times n}$ represent the space consisting of $m \times n$ complex matrices and the space consisting of $m \times n$ orthogonal complex matrices, respectively. For a vector $\mathbf{x}, \mathbf{x} \sim \mathbb{C N}(\boldsymbol{\mu}, \mathbf{R})$ represents that $\mathbf{x}$ follows a complex Gaussian distribution with mean $\boldsymbol{\mu}$ and covariance matrix $\mathbf{R}$; for a matrix $\mathbf{X}$, the notations $\mathbf{X}^{1 / 2}, \mathbf{X}^{H}$, and $\operatorname{Tr}(\mathbf{X})$ denote its square root, Hermitian transpose, and trace, respectively; besides, $\mathbf{I}_{m}$ is an $m \times m$ identity matrix, $\mathbf{I}_{m, n}$ with $n<m$ is created by selecting the first $n$ columns of $\mathbf{I}_{m}$, and $\mathbf{U}_{m}$ is an $m \times m$ unitary matrix. 


\section{The closed-loop UAV-enabled MIMO system model}

\subsection{The UAV MIMO channel}

As shown in Fig. 1, we consider a UAV-enabled MIMO system, where the base station (BS) and the UAV are equipped with $N_{T}$ and $N_{R}$ antennas, respectively [27, 28]. Both the BS and UAV are equipped with cache storage units. With the aid of cache, the UAV can store data conveniently for its own use or forward them to other UAVs/ground terminals at the next time. Figure 1a is the original system diagram, from which we see that there exists the line of sight (LOS) path between BS and UAV; due to the reflections of high buildings, trees, or others, there also exist non-LOS (NLOS) paths. Hence, the channel between BS and UAV is modeled as a Ricean fading channel.

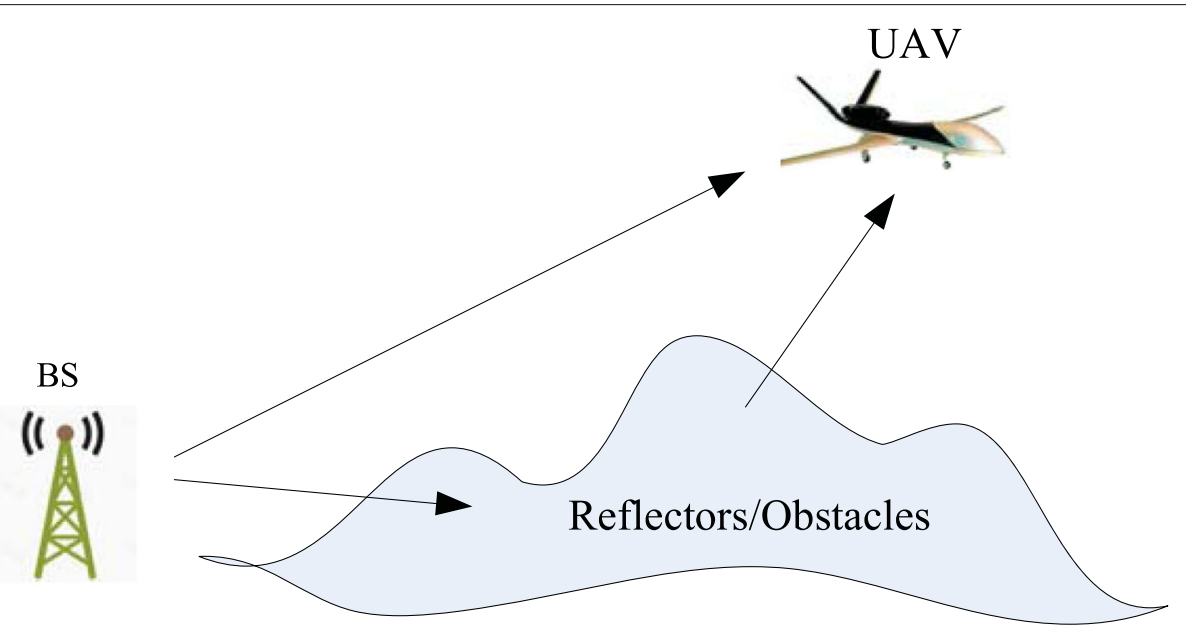

(a) The UAV MIMO system model

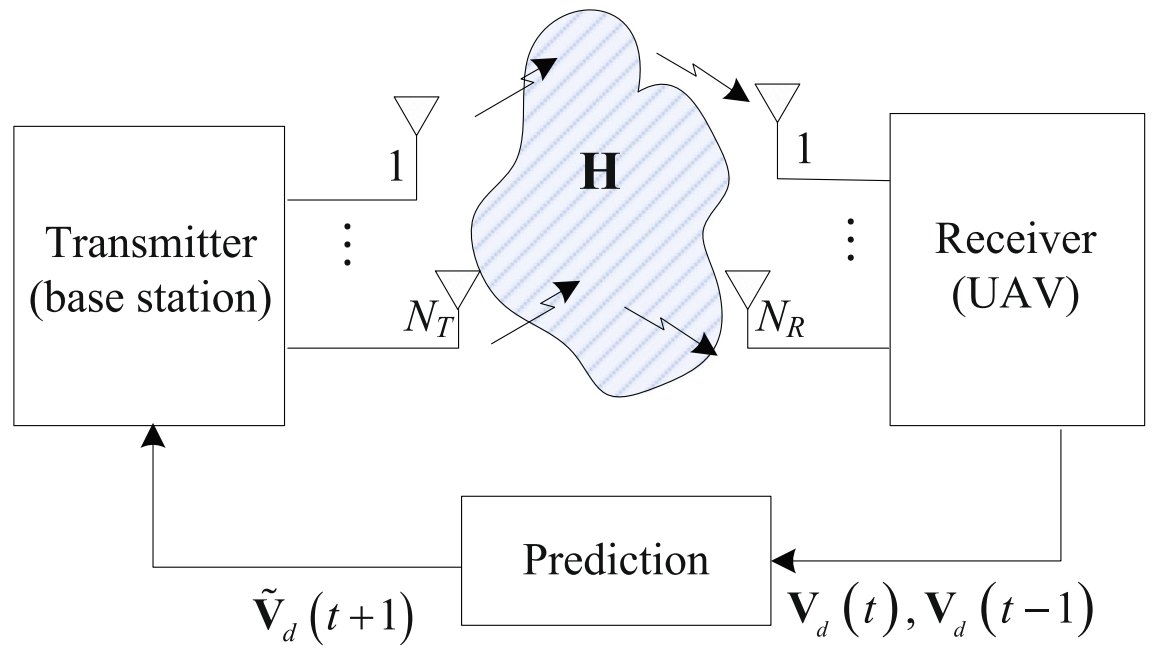

(b) The abstract equivalent system model

Fig. 1 MIMO system model with prediction feedback link 
For the UAV MIMO channel $\mathbf{H}(t) \in \mathbb{C}^{N_{R} \times N_{T}}$, we not only consider its time variation, but also the spatial correlation. In [29], a spatial-temporal correlated channel model was proposed; however, only transmitter correlation was considered. Herein, we also incorporate the receiver correlation of the channel. Consequently, the UAV MIMO channel is modeled as:

$$
\mathbf{H}(t)=\frac{\mathbf{H}_{\mathrm{NLOS}}(t)}{\sqrt{K_{\text {Rice }}+1}}+\sqrt{\frac{K_{\text {Rice }}}{K_{\text {Rice }}+1}} \mathbf{H}_{\mathrm{LOS}}(t) .
$$

In Eq. (1), the NLOS path $\mathbf{H}_{\mathrm{NLOS}}(t)$ is further expressed as:

$$
\mathbf{H}_{\mathrm{NLOS}}(t)=\boldsymbol{\theta}_{R}^{1 / 2} \mathbf{H}_{\omega}(t) \boldsymbol{\theta}_{T}^{1 / 2},
$$

where $\boldsymbol{\theta}_{T}$ and $\boldsymbol{\theta}_{R}$ denote the transmitter and receiver correlation matrix of the channel, respectively; $K_{\text {Rice }}$ is the Ricean factor. $\mathbf{H}_{\omega}(t) \triangleq\left(h_{i j}(t)\right) \in \mathbb{C}^{N_{R} \times N_{T}}$ and it is a random matrix that has i.i.d complex Gaussian random variables with zero mean and unit variance. The autocorrelation of each entry follows Jake's model and it is given by:

$$
\mathrm{E}\left[h_{i j}\left(t_{1}\right)\left(h_{i j}\left(t_{2}\right)\right)^{*}\right]=J_{0}\left(2 \pi f_{d}\left(t_{2}-t_{1}\right)\right),
$$

in which the function $J_{0}($.$) is the well-known Bessel function of the first kind, f_{d}$ is the Doppler shift, for the NLOS path, which is due to the motions of the UAV, scatters, etc.

Assuming that both BS and the UAV adopt the linear antenna array, the LOS path $\mathbf{H}_{\mathrm{LOS}}(t)$ is expressed as [30]:

$$
\begin{aligned}
& \mathbf{H}_{\mathrm{LOS}}(t)=e^{-j 2 \pi f^{\prime}{ }_{d} t} \mathbf{x}_{R}\left(\mathbf{x}_{T}\right)^{H}, \\
& \mathbf{x}_{R}=\left[\begin{array}{lll}
1 & e^{-j 2 \pi \cos (\alpha) d_{R} / \lambda} \cdots e^{-j 2 \pi\left(N_{R}-1\right) \cos (\alpha) d_{R} / \lambda}
\end{array}\right]^{T}, \\
& \mathbf{x}_{T}=\left[\begin{array}{lll}
1 & \left.e^{-j 2 \pi \cos (\beta) d_{T} / \lambda} \cdots e^{-j 2 \pi\left(N_{T}-1\right) \cos (\beta) d_{T} / \lambda}\right]^{T},
\end{array}\right.
\end{aligned}
$$

where $f_{d}^{\prime}$ is Doppler shift for the LOS path, which is due to the motion of the UAV; $d_{R}$ and $d_{T}$ are the antenna spacing at BS and the UAV, respectively; $\lambda$ is the wavelength; and $\alpha$ and $\beta$ are the angles of arrival and departure, respectively.

Note that the channel defined in Eq. (1) is assumed to be flat fading. If the paths are resolvable, we can incorporate the OFDM technique and transform the frequency selective channel into a flat fading one.

Remark: Concerning the angles of arrival and departure, the definitions are explained as follows. Take the angle of arrival as an example. As in Fig. 2, a plane electromagnetic wave arrives at the uniform linear antenna array. We adopt the definition of [30] and define $\alpha$ as the direction of arrival (DOA), i.e., the angle between the incident direction of the electromagnetic wave and the $x$-axis. Consequently, the time delay between the two adjacent antennas is $d_{R} \cos (\alpha) / \lambda$, which determines the form of the steering vector $\mathbf{x}_{R}$. Whereas in [15], the authors define $\alpha^{\prime}$ as the DOA. Consequently, the time delay between the two adjacent antennas is $d_{R} \sin \left(\alpha^{\prime}\right) / \lambda$. Clearly, the two definitions are equivalent since $\sin \left(\alpha^{\prime}\right)=\cos (\alpha)$. Besides, it is worth noting that the latter requires the assumption that the incident direction of the wave is only in the $x-y$ plane but the former does not require any assumptions. Therefore, we adopt the former definition.

\subsection{The system operation}

Before transmission, the user signal $\mathbf{x}(t) \in \mathbb{C}^{N_{s} \times 1}$, sent by the transmitter, is multiplied by a precoding matrix $\mathbf{W}(t) \in \mathbb{U}^{N_{T} \times N_{S}}$, where $t$ is the time index and $N_{S}$ is the number 


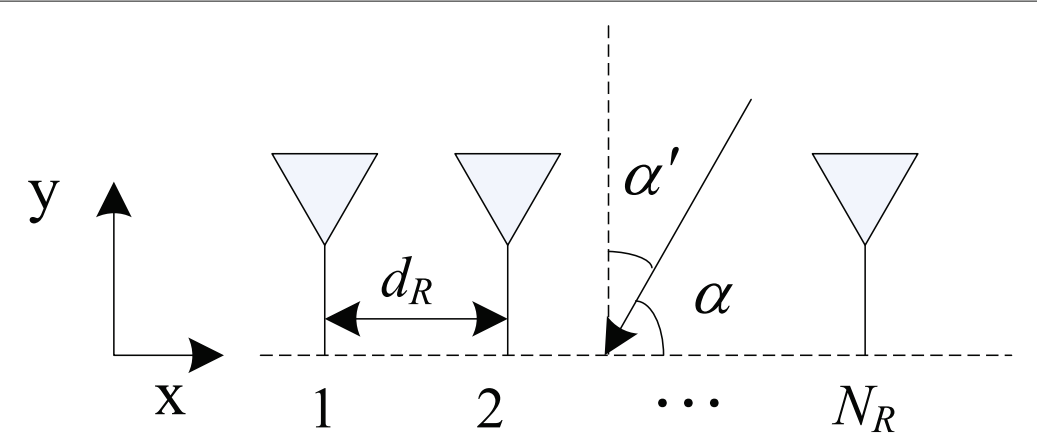

Fig. 2 The uniform linear antenna array and the arrival electromagnetic wave

of data streams. After passing through fading channels, the signal at the receiver can be formulated as:

$$
\mathbf{y}(t)=\mathbf{H}(t) \mathbf{W}(t) \mathbf{x}(t)+\mathbf{n}(t),
$$

in which $\mathbf{x}(t)$ has independent data streams with its autocorrelation function being $\mathrm{E}\left[\mathbf{x}(t) \mathbf{x}^{H}(t)\right]=\mathbf{I}_{N_{S}}$, and $\mathbf{n}(t) \sim \mathbb{C N}\left(\mathbf{0}, \sigma_{n}^{2} \mathbf{I}_{N_{R} \times N_{R}}\right)$ is the AWGN noise [31, 32], where the effect of noise on the communication systems can be found in [33, 34]. Notation $\mathbf{H}(t) \in \mathbb{C}^{N_{R} \times N_{T}}$ denotes the MIMO flat fading channel, where the fading scenarios can be found in [35-37].

Assume that the channel estimation is ideal and the receiver has perfect channel state information (CSI) [38-40], $\mathbf{H}(t)$. If the channel is quasi-static fading, which means that two adjacent channel matrices can be viewed as identical, i.e., $\mathbf{H}(t+1) \approx \mathbf{H}(t)$, the optimal precoder at time $(t+1)$ is given by:

$$
\mathbf{W}^{O P T}(t+1)=\mathbf{V}_{d}(t) \mathbf{U}_{d},
$$

where $\mathbf{U}_{d} \in \mathbb{U}^{N_{S} \times N_{S}}$ is an arbitrary unitary matrix and $\mathbf{V}_{d}(t) \in \mathbb{U}^{N_{T} \times N_{S}}$ is conducted by selecting the first $N_{S}$ columns of $\mathbf{V}(t)$, which comes from singular value decomposition (SVD) of $\mathbf{H}(t)$, expressed as:

$$
\mathbf{H}(t)=\mathbf{U}(t) \Lambda(t) \mathbf{V}^{H}(t) .
$$

The precoder expressed by (6) can not only maximize the system asymptotic capacity at high signal to noise ratio (SNR), but also minimize the mean square error of data detection.

However, if the channel is fast fading, the precoder at current time $t$ is no longer optimal for the next time $t+1$. In this case, as in Fig. 1b, we need a predictor, which produces a predicted precoder $\widetilde{\mathbf{V}}_{d}(t+1)$ for time $t+1$ by the use of $\mathbf{V}_{d}(t)$ and $\mathbf{V}_{d}(t-1)$, where the definition of $\mathbf{V}_{d}(t-1)$ is similar to $\mathbf{V}_{d}(t)$. Consequently, the precoder at time $t+1$ is given by:

$$
\mathbf{W}(t+1)=\widetilde{\mathbf{V}}_{d}(t+1) .
$$

Clearly, the smaller the distance between $\widetilde{\mathbf{V}}_{d}(t+1)$ and $\mathbf{V}_{d}(t+1)$, the better the prediction performance. We use the chordal distance to measure their distance, the details of which will be given in the next section. 
In addition, in the process of channel prediction, there is a key parameter termed as the step parameter. An aim of this article is to study the effect of various channel settings on the optimal step parameter as well as the expected chordal distance (error).

\section{Channel prediction precoding method}

\subsection{Preliminaries of the Grassmannian manifold}

Grassmannian manifold, denoted as $\mathbb{G}\left(N_{T}, N_{S}\right)$ with $N_{S}<N_{T}$, is important in mathematical differential geometry and has many engineering applications such as channel tracking [41, 42], image identification [43], and emotion recognition [44] in recent years. It is the set of all $N_{S}$-dimensional linear subspaces of an $n$-dimensional space. The corresponding definition is given by:

$$
\mathbb{G}\left(N_{T}, N_{S}\right)=\left\{[\mathbf{X}] \in \mathbb{C}^{N_{T} \times N_{S}}, \mathbf{X}^{H} \mathbf{X}=\mathbf{I}_{N_{S}}\right\},
$$

where $[\mathbf{X}]$ is an equivalent class that is a set defined by $[\mathbf{X}] \triangleq\left\{\mathbf{X U}_{N_{S}}: \mathbf{U}_{N_{S}}\right.$ is unitary $\}$. For the sake of convenience, we might as well write $\mathbf{X} \in \mathbb{G}\left(N_{T}, N_{S}\right)$, meaning that $\mathbf{X}$ is an equivalent class whose columns span the same $p$-dimensional subspace [16, 45]. For two points $\mathbf{X}_{1}, \mathbf{X}_{2} \in \mathbb{G}\left(N_{T}, N_{S}\right)$, the chordal distance is usually used to measure their distance and it is defined as [14, 23]:

$$
D\left(\mathbf{X}_{1}, \mathbf{X}_{2}\right)=\sqrt{N_{S}-\left\|\mathbf{X}_{1}{ }^{H} \mathbf{X}_{2}\right\|_{F}},
$$

where $\|\cdot\|_{F}$ denotes the Frobenius norm.

Besides, the concept of geodesic plays an important role in information geometry. The term geodesic comes from geodesy, the science of measuring the Earth. Initially, a geodesic was the shortest route between two points on the Earth's surface. Now, it is generalized and defined as the shortest route between two points of a manifold. The geodesic emanating from $\mathbf{X}_{1}$ to $\mathbf{X}_{2}$ is expressed as [41, 42]:

$$
\mathbf{X}(s)=\mathbf{Q}_{1} \exp (\mathbf{B} s) \mathbf{I}_{N_{T}, N_{S}}, 0 \leq s \leq 1 .
$$

where $\mathbf{Q}_{1} \in \mathbb{U}^{N_{T} \times N_{T}}=\left(\mathbf{X}_{1} \mathbf{X}_{1}^{\perp}\right)$, and $\mathbf{X}_{1}^{\perp} \in \mathbb{U}^{N_{T} \times\left(N_{T}-N_{S}\right)}$ is some orthogonal basis of the orthogonal complement of $\mathbf{X}_{1}$. The matrix $\mathbf{B} \in \mathbb{C}^{N_{T} \times N_{T}}$ is skew Hermitian, and it has the following form:

$$
\mathbf{B}=\left(\begin{array}{cc}
\mathbf{0} & -\mathbf{A}^{H} \\
\mathbf{A} & \mathbf{0}
\end{array}\right),
$$

where $\mathbf{A} \in \mathbb{C}^{\left(N_{T}-N_{S}\right) \times N_{S}}$ is referred to as the velocity matrix. The matrix $\mathbf{A}$ can be further written as:

$$
\mathbf{A}=\mathbf{U}_{2} \Phi \mathbf{U}_{1}^{H},
$$

where $\Phi$ is diagonal with its elements $\varphi_{i}, 1 \leq i \leq N_{S}$. The matrices $\mathbf{U}_{1} \in \mathbb{U}^{N_{S} \times N_{S}}$ and $\mathbf{U}_{2} \in \mathbb{U}^{\left(N_{T}-N_{S}\right) \times N_{S}}$ come from the cosine-sine decomposition of a specially constructed matrix [41, 42]:

$$
\left(\begin{array}{c}
\mathbf{X}_{1}^{H} \mathbf{X}_{2} \\
\left(\mathbf{X}_{1}^{\perp}\right)^{H} \mathbf{X}_{2}
\end{array}\right)=\left(\begin{array}{cc}
\mathbf{U}_{1} & \mathbf{0} \\
\mathbf{0} & \mathbf{U}_{2}
\end{array}\right)\left(\begin{array}{l}
\mathbf{C} \\
\mathbf{S}
\end{array}\right) \mathbf{V}_{1}^{H},
$$

in which $\mathbf{C}$ is diagonal with its entries $\cos \varphi_{i}$ on the diagonal; $\mathbf{S}$ is also diagonal with its entries $\sin \varphi_{i}$, on the diagonal, $1 \leq i \leq N_{S}$; and $\mathbf{V}_{1}$ is the right singular matrix of the SVD of $\mathbf{X}_{1}^{H} \mathbf{X}_{2}$, which can also be inferred from Eq. (14). 
With Eqs. (12)-(14), the key matrix B will be created and the geodesic Eq. 11 can be finally given afterwards. According to the original definition of $\mathrm{X}(\mathrm{s})$, we have the following corollary:

$$
\mathbf{X}(1)=\mathbf{Q}_{1} \exp (\mathbf{B}) \mathbf{I}_{N_{T}, N_{S}}=\mathbf{X}_{2}
$$

We believe that the above is important since it can verify the correctness of the geodesic equation; however, it was not proved in $[41,42]$. Herein, we supply a proof which is shown in the Appendix.

\subsection{Review of the conventional channel prediction method [41]}

The matrix $\mathbf{V}_{d}(t)$ can be viewed as a point of Grassmannian manifold. It is reasonable to assume that the matrix $\mathbf{V}_{d}(t)$ moves or changes along the geodesic within a short period of time. The conventional prediction obtains the next point mainly by two steps: (1) parallel transport the tangent matrix at $\mathbf{V}_{d}(t-1)$ and (2) construct a new geodesic for prediction.

First, given two points $\mathbf{V}_{d}(t)$ and $\mathbf{V}_{d}(t-1)$, the tangent matrix at the point $\mathbf{V}_{d}(t-1)$, in the direction of the next point $\mathbf{V}_{d}(t)$, is given by [41]:

$$
\Delta(t-1)=\mathbf{V}_{d}^{\perp}(t-1) \mathbf{A}(t-1),
$$

where $\mathbf{V}_{d}^{\perp}(t-1) \in \mathbb{U}^{N_{T} \times\left(N_{T}-N_{S}\right)}$ is some orthogonal basis of orthogonal complement of $\mathbf{V}_{d}(t-1), \mathbf{A}(t-1) \in \mathbb{C}^{\left(N_{T}-N_{S}\right) \times N_{S}}$ is the velocity matrix, and other details are easy to deduce by referring to the previous subsection.

Then, we transport the tangent matrix $\Delta(t-1) \in \mathbb{C}^{N_{T} \times N_{S}}$ parallel along the geodesic between $\mathbf{V}_{d}(t)$ and $\mathbf{V}_{d}(t-1)$, and obtain a new tangent matrix [45]:

$$
\widetilde{\Delta}\left(t, s_{0}\right)=\left[\begin{array}{c}
\mathbf{I}_{N_{T}}-\mathbf{U}_{F} \mathbf{U}_{F}^{H}+\mathbf{V}_{d}(t-1) \mathbf{V}_{F} \sin \left(\Lambda_{F} s_{0}\right) \mathbf{U}_{F}^{H} \\
+\mathbf{U}_{F} \cos \left(\Lambda_{F} s_{0}\right) \mathbf{U}_{F}^{H}
\end{array}\right] \Delta(t-1),
$$

where $0 \leq s_{0} \leq 1$ is the step parameter and $\mathbf{U}_{F} \Lambda_{F} \mathbf{V}_{F}^{H}$ is the compact SVD of the matrix $\Delta(t-1)$. It is clear that $\widetilde{\Delta}(t, 0)=\Delta(t-1)$ and $\widetilde{\Delta}(t, 1)$ is the tangent matrix at the point $\mathbf{V}_{d}$; we shall transport the tangent matrix $\Delta(t-1)$ parallel from $\mathbf{V}_{d}(t-1)$ to $\mathbf{V}_{d}(t)$, which means $s_{0}=1$.

Finally, the predictive point on the manifold at time $t+1, \overline{\mathbf{V}}_{d}^{\text {Conv }}(t, s)$, can be derived as $[23,41,45]$ :

$$
\overline{\mathbf{V}}_{d}^{\text {Conv }}(t, s)=\mathbf{V}_{d}(t) \mathbf{V}_{E} \cos \left(\Lambda_{E} s\right) \mathbf{V}_{E}^{H}+\mathbf{U}_{E} \sin \left(\Lambda_{E} s\right) \mathbf{V}_{E}^{H},
$$

where $\mathbf{U}_{E} \Lambda_{E} \mathbf{V}_{E}^{H}$ is the compact SVD of the matrix $\widetilde{\Delta}(t, 1)$ and $s$ is the step parameter which can be further optimized. The optimal step $s_{O P T}$ can be found by solving the following equation:

$$
s_{O P T}=\underset{s}{\arg \min } \mathrm{E}\left[D\left(\overline{\mathbf{V}}_{d}^{\text {Conv }}(t, s), \mathbf{V}_{d}(t+1)\right)\right] .
$$

Once the optimal step is found, we substitute it into (18) to obtain $\widetilde{\mathbf{V}}_{d}^{\text {Conv }}(t+1) \triangleq$ $\overline{\mathbf{V}}_{d}^{\text {Conv }}\left(t, s_{O P T}\right)$.

\subsection{The proposed channel prediction method}

In this subsection, we propose a novel channel prediction method, in which the next point is predicted by use of the geodesic from $\mathbf{V}_{d}(t-1)$ to $\mathbf{V}_{d}(t)$ directly. First, 
according to the previous subsection $\mathrm{A}$, the geodesic emanating from $\mathbf{V}_{d}(t-1)$ to $\mathbf{V}_{d}(t)$ is expressed as:

$$
\mathbf{F}(t-1, s)=\mathbf{Q}_{1}(t-1) \exp [\mathbf{B}(t-1) s] \mathbf{I}_{N_{T}, N_{s}}
$$

where $\mathbf{Q}_{1}(t-1) \in \mathbb{U}^{N_{T} \times N_{T}}=\left(\mathbf{V}_{d}(t-1) \mathbf{V}_{d}^{\perp}(t-1)\right)$; $\mathbf{V}_{d}^{\perp}(t-1)$ and $\mathbf{B}(t-1)$ have similar definitions in the previous subsection A. Clearly, $\mathbf{F}(t-1,0)=\mathbf{V}_{d}(t-1)$ and $\mathbf{F}(t-1,1)=\mathbf{V}_{d}(t)$.

Second, the predictive point on the manifold at time $t+1$ is given by:

$$
\mathbf{F}(t-1, s+1)=\mathbf{Q}_{1}(t-1) \exp [(s+1) \mathbf{B}(t-1)] \mathbf{I}_{N_{T}, N_{s}}, 0 \leq s \leq 1 .
$$

Similarly, the optimal step $s_{O P T}$ in the above formula can be found by solving Eq. (19). Note that the function $\overline{\mathbf{V}}_{d}^{\text {Conv }}(t, s)$ in Eq. (19) shall be replaced with $\mathbf{F}(t-1, s+1)$. Once it is found, the precoding matrix or predictive precoder is solved by $\widetilde{\mathbf{V}}_{d}^{\text {Prop }}(t+1) \triangleq$ $\mathbf{F}\left(t-1, s_{O P T}+1\right)$.

The complete prediction process is summarized by Algorithm 1 .

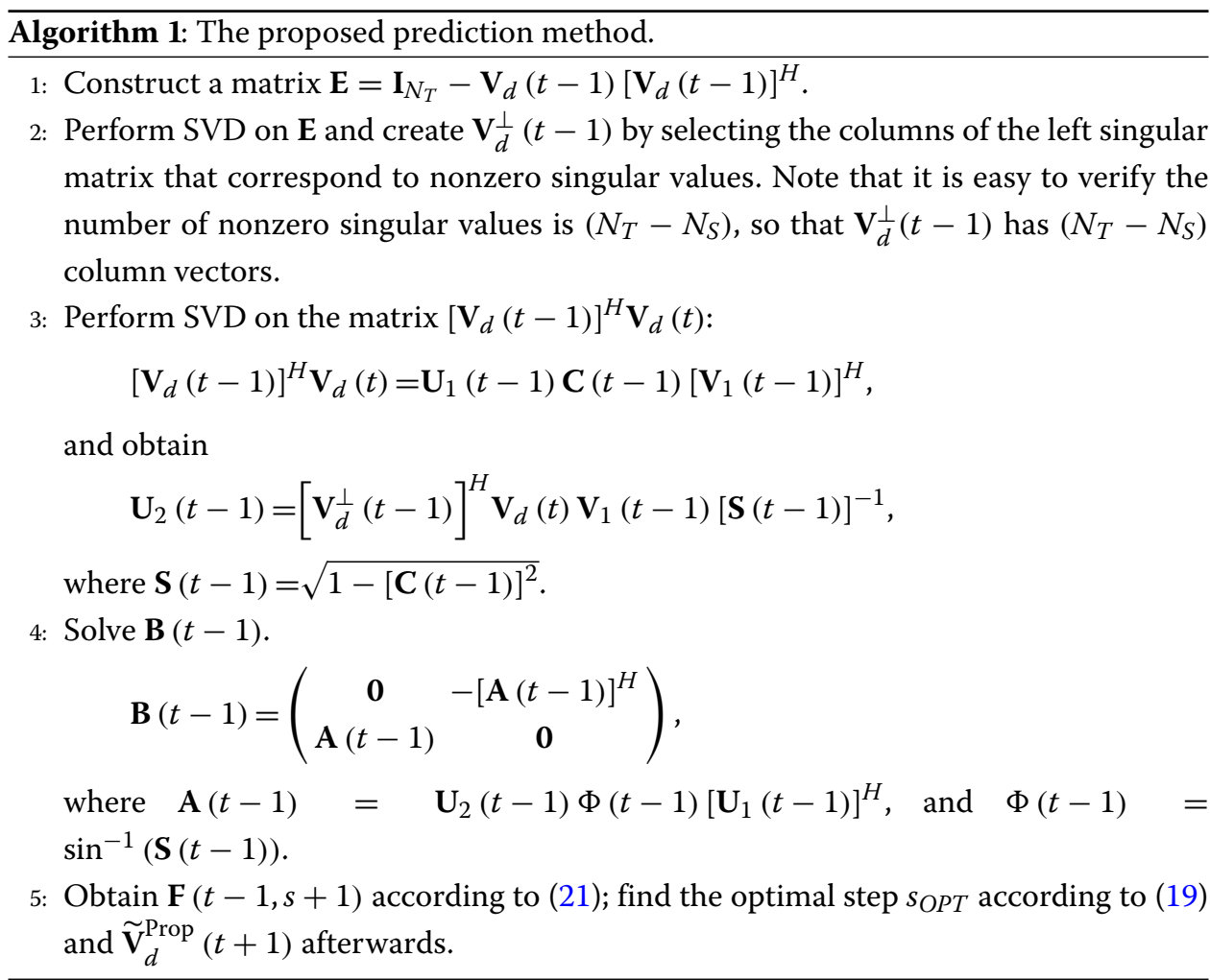

Note that in the calculation of the matrix function $\exp [\mathbf{B}(t-1) s]$, we shall use the following matrix series [46]:

$$
\exp [\mathbf{B}(t-1) s]=\sum_{i}[\mathbf{B}(t-1) s]^{i} / i !
$$

\subsection{Computational complexity}

Usually, the number of floating point flops is used to measure of the complexity of an algorithm. We define a flop as one floating point operation and it has computational 
complexity $O(1)$. Note that the matrix product $\mathbf{X}_{1} \mathbf{X}_{2}$ requires $O(m n k)$ flops, where $\mathbf{X}_{1} \in \mathbb{C}^{m \times n}$ and $\mathbf{X}_{2} \in \mathbb{C}^{n \times k}$; for an $n \times n$ matrix, both its inverse and eigen-decomposition operations require $O\left(n^{3}\right)$ flops; for an $m \times n$ matrix with $m \geq n$, the complexity of its singular value decomposition (SVD) is $O\left(m^{2} n\right)$ if the complete left singular matrix is needed; otherwise, it is $O\left(m n^{2}\right)$ [47] .

To begin with, we analyze the computational complexity of the proposed method. Initially, the two points $\mathbf{V}_{d}(t-1)$ and $\mathbf{V}_{d}(t)$ are generated from the SVD of $\mathbf{H}(t-1)$ and $\mathbf{H}(t)$, respectively. Since the left singular matrices are not needed, this operation has complexity of $O\left(n_{\max } n_{\text {min }}^{2}\right)$, where $n_{\max }=\max \left(N_{T}, N_{R}\right), n_{\min }=\min \left(N_{T}, N_{R}\right)$. Then, it is found that step 1 requires $O\left(N_{T}^{2} N_{S}\right)$ flops and step 2 requires $O\left(N_{T}^{3}\right)$ flops; step 3 requires $\left.O\left(N_{S}^{2} N_{T}\right)+O\left(N_{S}^{3}\right)+O\left[\left(N_{T}-N_{S}\right) N_{T} N_{S}\right)\right]=O\left(N_{T}^{2} N_{S}\right)$ flops, since $N_{S} \leq N_{T}$; and step 4 requires $\left.O\left(N_{T}-N_{S}\right) N_{S}^{2}\right)$ flops. For the calculation of $\exp [\mathbf{B}(t-1) s]$ in step 5, by using the techniques in the Appendix, we have that:

$$
\exp [\mathbf{B}(t-1) s]=\left(\begin{array}{ll}
\mathbf{U}_{1}(t-1) \cos (\Phi s) \mathbf{U}_{1}^{H}(t-1) & -\mathbf{U}_{1}(t-1) \sin (\Phi s) \mathbf{U}_{2}^{H}(t-1) \\
\mathbf{U}_{2}(t-1) \sin (\Phi s) \mathbf{U}_{1}^{H}(t-1) & \mathbf{U}_{2}(t-1) \cos (\Phi s) \mathbf{U}_{2}^{H}(t-1)
\end{array}\right) .
$$

With the above, given $s$, solving $\mathbf{F}(t-1, s)$ has complexity of $O\left(N_{T} N_{S}^{2}+N_{T}^{2} N_{S}\right)=$ $O\left(N_{T}^{2} N_{S}\right)$. Assuming that the minimal search interval that stands for the accuracy of $s_{O P T}$ is $\xi, 1 / \xi$ times of calculating $\mathbf{F}(t-1, s)$ are required to find $s_{O P T}$. Hence, step 5 has complexity of $O\left(N_{T}^{2} N_{S} / \xi\right)$. In summary, the complexity of the proposed method is $O\left(n_{\max } n_{\min }^{2}\right)+O\left(N_{T}^{3}\right)+O\left(N_{T}^{2} N_{S} / \xi\right)=O\left[\left(n_{\max } n_{\text {min }}^{2}\right)+N_{T}^{2}\left(N_{T}+N_{S} / \xi\right)\right]$, where $n_{\text {max }}=\max \left(N_{T}, N_{R}\right), n_{\text {min }}=\min \left(N_{T}, N_{R}\right)$.

Then, we analyze the complexity of the conventional method briefly. Initially, we also need the two points $\mathbf{V}_{d}(t-1)$ and $\mathbf{V}_{d}(t)$. In step 1 , to generate $\mathbf{V}_{d}^{\perp}(t-1)$, assume that the same method as in the subsection $C$ is used. Further, we note that for an $m \times n$ matrix with $m \geq n$, the compact SVD has complexity of $O\left(m n^{2}\right)$ because the full right singular matrix is not needed. Paying attention to the above points, it is easy to find that the complexity of the conventional method is the same as the proposed method.

\section{Results and discussion}

In this section, computer simulation is deployed to investigate the performance of the prediction algorithm. The UAV MIMO channel samples are generated according to the channel model in Section 2. Specifically, a $4 \times 4$ UAV MIMO system with $N_{S}=2$ is adopted. For the transmitter and receiver correlation matrices, $\boldsymbol{\theta}_{T}$ and $\boldsymbol{\theta}_{\boldsymbol{R}}$, the well-known exponential correlation model is adopted [48] , in which the $(i, j)$ th entry of the correlation matrix is $\rho^{|i-j|}$, and the constant $\rho$ is the spatial correlation coefficient. We further denote the correlated coefficients at the transmitter and receiver as $\rho_{T}$ and $\rho_{R}$, respectively. The angles of arrival and departure $\alpha$ and $\beta$ are set to $30^{\circ}$ and $20^{\circ}$, respectively; the antenna spacing $d_{T}=d_{R}=\lambda$. Besides, the Doppler shifts for the LOS path and NLOS path are set to be identical, i.e., $f_{d}^{\prime}=f_{d}$.

\subsection{Performance comparison between the proposed method and the conventional one}

Figure 3 compares the chordal error performance of the conventional method with that of the proposed one, where $\rho_{T}=0.2$ and $\rho_{R}=0.3$. Two cases are considered: $f_{d} T=$ 0.02 and $K_{\text {Rice }}=0 \mathrm{~dB}$ for case 1 and $f_{d} T=0.05$ and $K_{\text {Rice }}=10 \mathrm{~dB}$ for case 2, where 


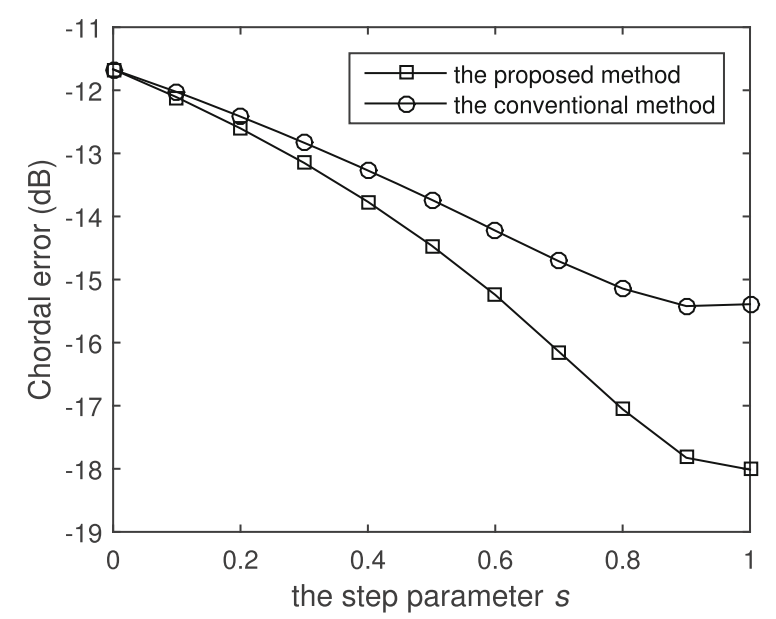

(a) case 1

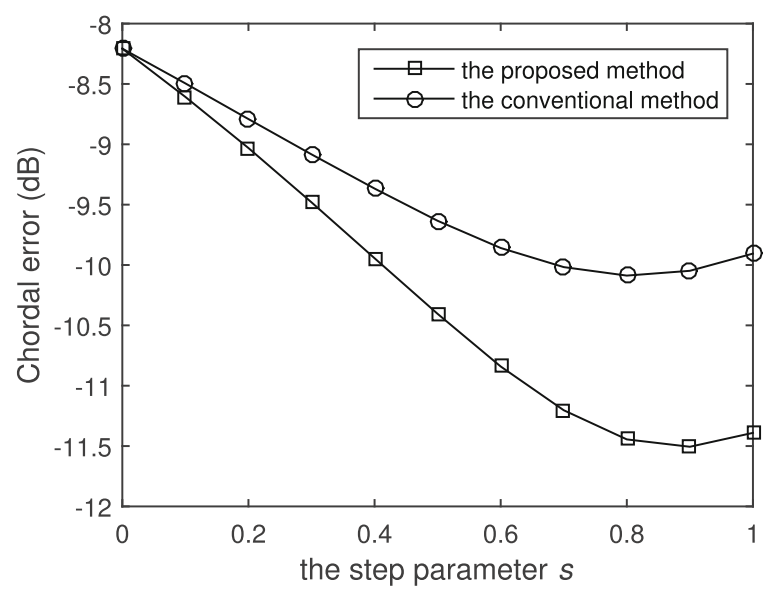

(b) case 2

Fig. 3 Performance comparison between the conventional method and the proposed one

$f_{d} T$ is the normalized Doppler shift, $f_{d}$ is the Doppler shift, and $T$ is the sample interval. Observe that given $s>0$, the chordal error for the proposed method is always less than that for the conventional method. For case 1 in Fig. 3a, the minimum chordal error for the conventional method is about $-15.42 \mathrm{~dB}$ at $s=0.9$; the minimum chordal error for the proposed one is $-18.01 \mathrm{~dB}$ at $s=1.0$ and it is less than $-15.42 \mathrm{~dB}$. Therefore, the proposed method is superior to the conventional method. Similar phenomena can be observed in Fig. 3b.

Figure 4 compares two methods with different values of normalized Doppler shift $f_{d} T$, in which $\rho_{T}=0.2, \rho_{R}=0.3$, and $K_{\text {Rice }}=0 \mathrm{~dB}$. Observe that for both methods, given the step parameter $s$, increasing $f_{d} T$ results in increasing chordal error. Increasing $f_{d} T$ also has the optimal step parameter $s_{O P T}$ decrease. For instance, for the proposed method, the optimal steps are about 1, 0.9, and 0.6, for $f_{d} T=0.01,0.05$, and 0.1, respectively. Similar to Fig. 3, the proposed method has better performance than the conventional method, no matter whether the normalized Doppler shift is large or not. 


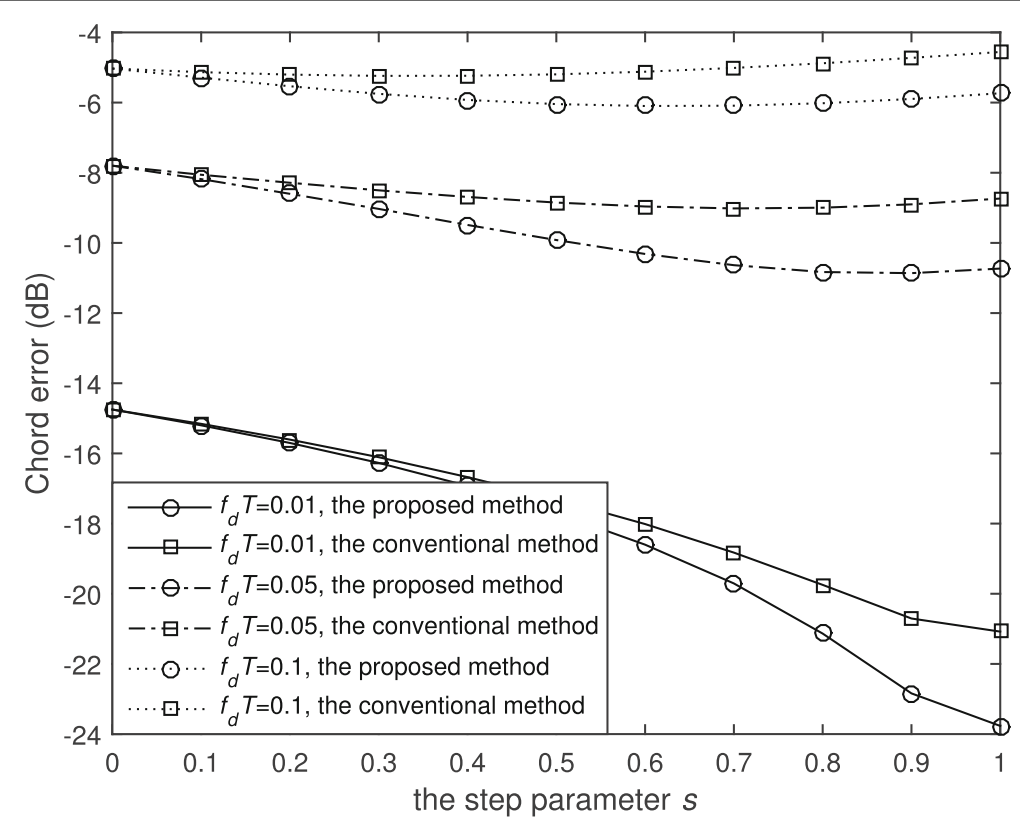

Fig. 4 Two methods' performance comparison with different values of normalized Doppler shift $f_{d} T$

\subsection{The effects of a few key system parameters}

Figure 5 shows the optimal step parameter $s_{O P T}$ with different normalized Doppler shifts. The system settings are consistent with those in Fig. 4. Observe that for increasing normalized Doppler shift, $s_{O P T}$ decreases from 1 to 0.6 gradually. The optimal step $s_{O P T}$ is found by solving Eq. (19), where the searching step is set to 0.1, and hence, the curve shows a stepwise downward trend.

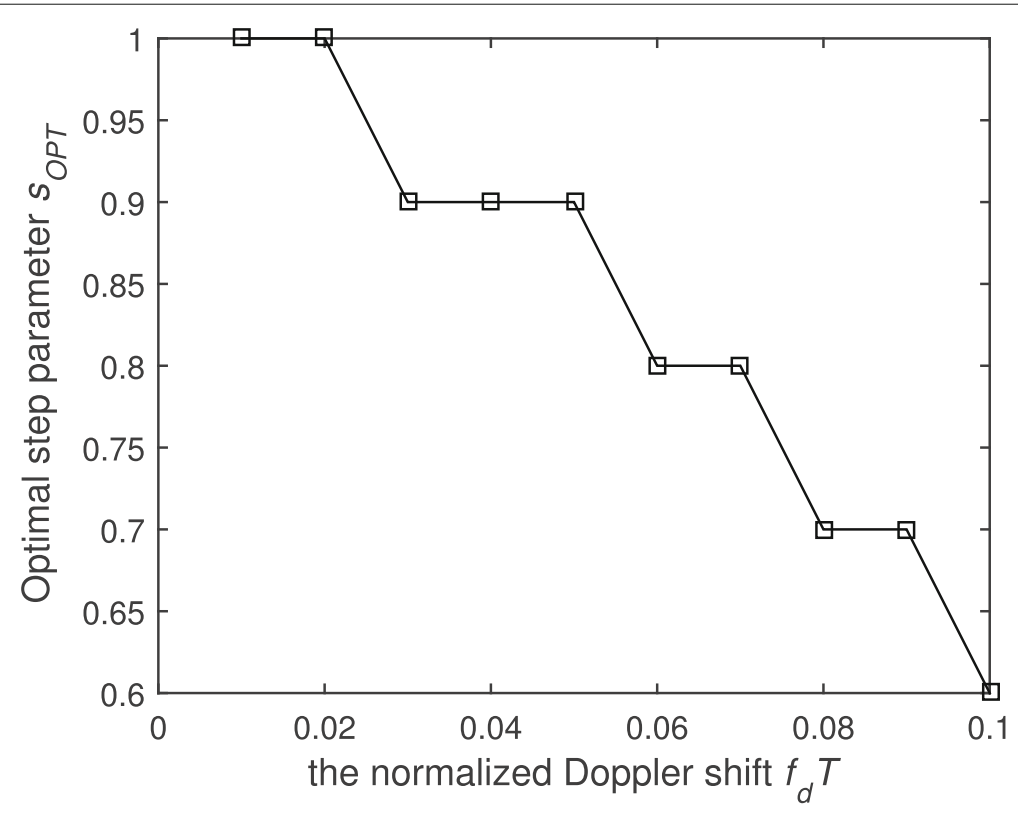

Fig. 5 The optimal step sOPT with different values of normalized Doppler shift $f_{d} T$ 


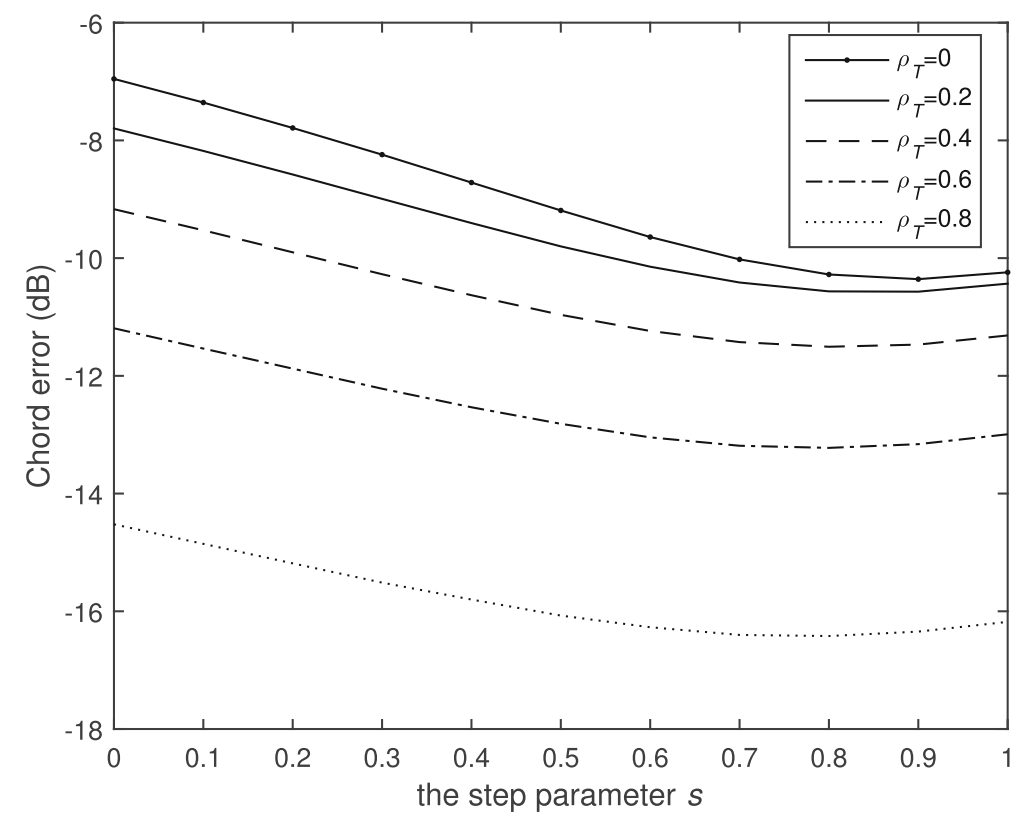

Fig. 6 The effect of transmitter correlated coefficient $\rho_{T}$ on the chordal error

Figures 6 and 7 present the effects of $\rho_{T}$ and $\rho_{R}$ on the chordal error, respectively. For both figures, $f_{d} T=0.05$ and $K_{\text {Rice }}=0 \mathrm{~dB}$. Further, we set $\rho_{R}=0.2$ for Fig. 6 and $\rho_{T}=0.2$ for Fig. 5, respectively. From Fig. 6, we observe that given the step parameter $s$, increasing $\rho_{T}$ will decrease the chordal error and also decrease the optimal $s$. For instance, given $s$ $=0.5$, the chordal errors for $\rho_{T}=0,0.2,0.4,0.6$, and 0.8 are $-9.2 \mathrm{~dB},-9.8 \mathrm{~dB},-11.0 \mathrm{~dB}$, $-12.8 \mathrm{~dB}$, and $-15.8 \mathrm{~dB}$, respectively; the optimal $s$ decreases from 0.9 to 0.8 slightly when increasing $\rho_{T}$ from 0 to 0.8 . In Fig. 7 , increasing the correlation coefficient also reduces the chordal error, but conversely, the optimal $s$ increases from 0.8 to 0.9 slightly with increasing $\rho_{R}$. Therefore, these results indicate that both $\rho_{T}$ and $\rho_{R}$ are negatively related to the chordal error; $\rho_{T}$ is slightly negatively related to the optimal step parameter, whereas the opposite is true for $\rho_{R}$.

Figure 8 depicts the effect of the Ricean factor $K_{\text {Rice }}$ on the chordal error, where $f_{d} T=0.05, \rho_{T}=0.2$, and $\rho_{R}=0.3$. Observe that given $s$, larger $K_{\text {Rice }}$ results in smaller chordal error. This is possibly because that the increase of $K_{\text {Rice }}$ reduces the randomness of the UAV channel variation and tracking the LOS path is easier than the NLOS path. In addition, the optimal step $s_{O P T}$ is about 0.9 and nearly unaffected by $K_{\text {Rice }}$.

\section{Conclusions}

This article studies the problem of channel predictive precoding in UAV MIMO systems. A novel predictive precoding method that is based on the geodesic in the Grassmannia manifold is proposed. Instead of transporting the tangent vector parallel along the geodesic, the proposed method predicts the next point by use of the geodesic equation directly. We compare the proposed method with the conventional one by simulation and analyze their computational complexity. Besides, the effects of various system parameters, including time and spatial correlated coefficients and the Ricean factor, on the chordal error of the predictor are also thoroughly investigated. 


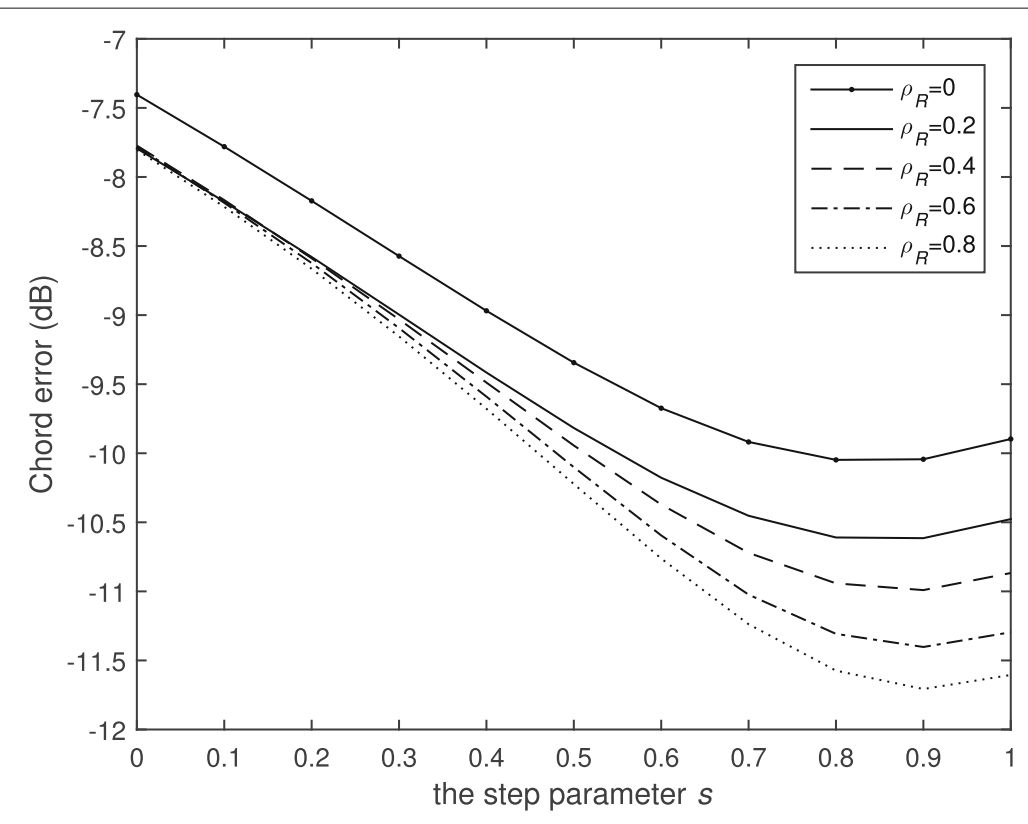

Fig. 7 The effect of receiver correlated coefficient $\rho_{R}$ on the chordal error

The results reveal the following. First, the normalized Doppler shift is positively related to the chordal error, whereas the transmitter and receiver correlation coefficients and the Ricean factor are negatively related to the chordal error. Second, the normalized Doppler shift is negatively related to the optimal step parameter; the transmitter correlation coefficient is slightly negatively related to the optimal step parameter, whereas the opposite is true for the receiver correlation coefficient. Third, with the same computational complexity, the proposed predictive algorithm is superior to the conventional method in terms of

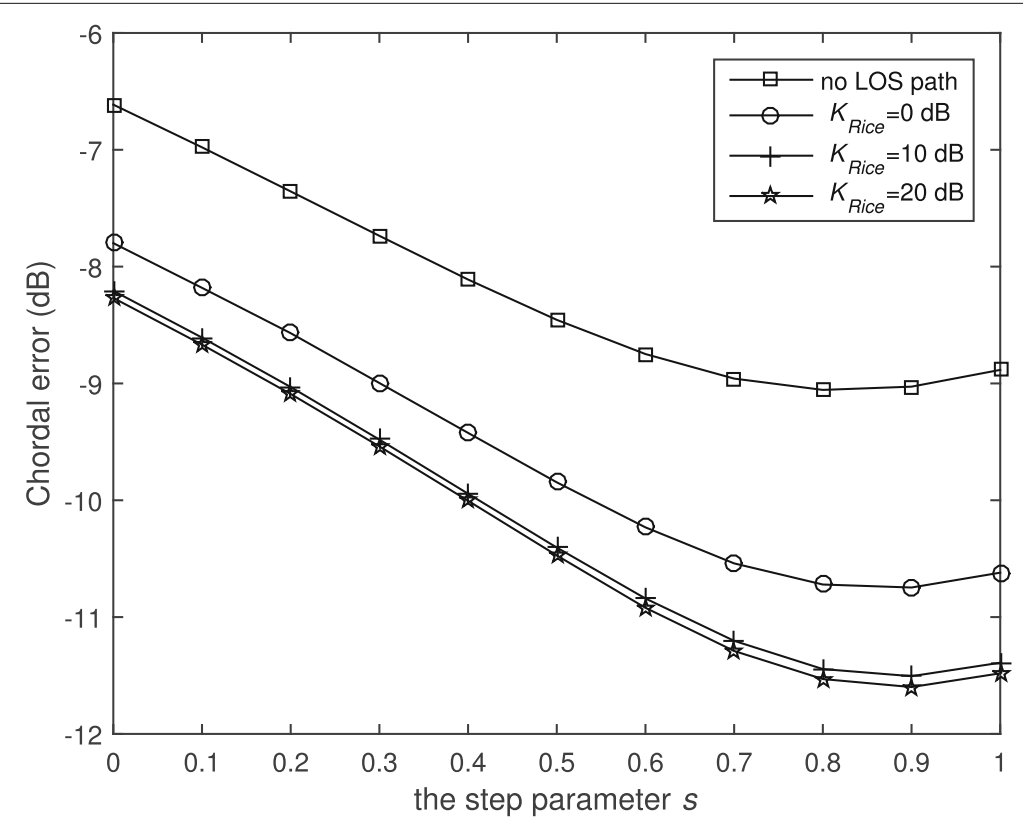

Fig. 8 The effect of the Ricean factor $K_{\text {Rice }}$ on the chordal error 
the channel predictive error-the channel chordal error. The above research results will provide a reference for possible system implementation in the future.

A further study is to investigate the effect of channel estimation errors on the predictor, where the two channel estimates include the previous and current estimates which are not assumed to be ideal. How to design a robust predictor is worthy of researching. Another possible study is to incorporate the codebook quantizer into the predictor design.

\section{Appendix}

Proof of Eq. (15)

First, the matrix function $\exp (\mathbf{B})$ can be expanded as:

$$
\begin{aligned}
\exp (\mathbf{B}) & =\sum_{i} \mathbf{B}^{i} / i ! \\
& =\mathbf{I}_{N}+\left(\begin{array}{cc}
\mathbf{0} & -\mathbf{U}_{1} \Phi \mathbf{U}_{2}^{H} \\
\mathbf{U}_{2} \Phi \mathbf{U}_{1}^{H} & \mathbf{0}
\end{array}\right) / 1 !+\left(\begin{array}{cc}
-\mathbf{U}_{1} \Phi^{2} \mathbf{U}_{1}^{H} & \mathbf{0} \\
\mathbf{0} & -\mathbf{U}_{2} \Phi^{2} \mathbf{U}_{2}^{H}
\end{array}\right) / 2 ! \\
& +\left(\begin{array}{cc}
\mathbf{0} & \mathbf{U}_{1} \Phi^{3} \mathbf{U}_{2}^{H} \\
-\mathbf{U}_{2} \Phi^{3} \mathbf{U}_{1}^{H} & \mathbf{0}
\end{array}\right) / 3 !+\left(\begin{array}{cc}
\mathbf{U}_{1} \Phi^{4} \mathbf{U}_{1}^{H} & \mathbf{0} \\
\mathbf{0} & \mathbf{U}_{2} \Phi^{4} \mathbf{U}_{2}^{H}
\end{array}\right) / 4 ! \\
& +\left(\begin{array}{cc}
\mathbf{0} & -\mathbf{U}_{1} \Phi^{5} \mathbf{U}_{2}^{H} \\
\mathbf{U}_{2} \Phi^{5} \mathbf{U}_{1}^{H} & \mathbf{0}
\end{array}\right) / 5 !+\ldots
\end{aligned}
$$

Since

$$
\sin \Phi=\sum_{i=1}^{+\infty}(-1)^{i} \Phi^{2 i+1} /(2 i+1) !
$$

and

$$
\cos \Phi=\sum_{i=0}^{+\infty}(-1)^{i} \Phi^{2 i} /(2 i) !, \quad \quad(A-3)
$$

the formula $(A-1)$ can be simplified as:

$$
\exp (\mathbf{B})=\left(\begin{array}{cc}
\mathbf{0} & -\mathbf{U}_{1} \sin \Phi \mathbf{U}_{2}^{H} \\
\mathbf{U}_{2} \sin \Phi \mathbf{U}_{1}^{H} & \mathbf{0}
\end{array}\right)+\left(\begin{array}{cc}
\mathbf{U}_{1} \cos \Phi \mathbf{U}_{1}^{H} & \mathbf{0} \\
\mathbf{0} & \mathbf{U}_{2} \cos \Phi \mathbf{U}_{2}^{H}
\end{array}\right) . \quad(A-4)
$$

Then, we have:

$$
\begin{aligned}
& \mathbf{X}(1)=\mathbf{Q}_{1} \exp (\mathbf{B}) \mathbf{I}_{N_{T}, N_{S}} \\
& =\mathbf{Q}_{1}\left\{\left(\begin{array}{cc}
\mathbf{U}_{1} \cos \Phi \mathbf{U}_{1}^{H} & \mathbf{0} \\
\mathbf{0} & \mathbf{U}_{2} \cos \Phi \mathbf{U}_{2}^{H}
\end{array}\right)+\left(\begin{array}{cc}
\mathbf{0} & -\mathbf{U}_{1} \sin \Phi \mathbf{U}_{2}^{H} \\
\mathbf{U}_{2} \sin \Phi \mathbf{U}_{1}^{H} & \mathbf{0}
\end{array}\right)\right\} \mathbf{I}_{N_{T}, N_{S}} \\
& =\left(\mathbf{X}_{1} \mathbf{X}_{1}^{\perp}\right)\left\{\left(\begin{array}{cc}
\mathbf{U}_{1} \cos \Phi \mathbf{U}_{1}^{H} & \mathbf{0} \\
\mathbf{0} & \mathbf{U}_{2} \cos \Phi \mathbf{U}_{2}^{H}
\end{array}\right)+\left(\begin{array}{cc}
\mathbf{0} & -\mathbf{U}_{1} \sin \Phi \mathbf{U}_{2}^{H} \\
\mathbf{U}_{2} \sin \Phi \mathbf{U}_{1}^{H} & \mathbf{0}
\end{array}\right)\right\} \mathbf{I}_{N_{T}, N_{S}} \quad(A-5) \\
& =\left[\left(\mathbf{X}_{1} \mathbf{U}_{1} \cos \Phi \mathbf{U}_{1}^{H} \mathbf{X}_{1}^{\perp} \mathbf{U}_{2} \cos \Phi \mathbf{U}_{2}^{H}\right)+\left(\mathbf{X}_{1}^{\perp} \mathbf{U}_{2} \sin \Phi \mathbf{U}_{1}^{H}-\mathbf{X}_{1} \mathbf{U}_{1} \sin \Phi \mathbf{U}_{2}^{H}\right)\right] \mathbf{I}_{N_{T}, N_{S}} \\
& =\mathbf{X}_{1} \mathbf{U}_{1} \cos \Phi \mathbf{U}_{1}^{H}+\mathbf{X}_{1}^{\perp} \mathbf{U}_{2} \sin \Phi \mathbf{U}_{1}^{H}
\end{aligned}
$$


Further manipulation of $(A-5)$ results in:

$$
\begin{aligned}
& \mathbf{X}(1)=\left(\begin{array}{ll}
\mathbf{X}_{1} & \mathbf{X}_{1}^{\perp}
\end{array}\right)\left(\begin{array}{cc}
\mathbf{U}_{1} & \mathbf{0} \\
\mathbf{0} & \mathbf{U}_{2}
\end{array}\right)\left(\begin{array}{c}
\cos \Phi \\
\sin \Phi
\end{array}\right) \mathbf{U}_{1}^{H} \\
& =\left(\begin{array}{ll}
\mathbf{X}_{1} & \mathbf{X}_{1}^{\perp}
\end{array}\right)\left(\begin{array}{cc}
\mathbf{U}_{1} & \mathbf{0} \\
\mathbf{0} & \mathbf{U}_{2}
\end{array}\right)\left(\begin{array}{l}
\mathbf{C} \\
\mathbf{S}
\end{array}\right) \mathbf{U}_{1}^{H} \\
& =\left(\begin{array}{ll}
\mathbf{X}_{1} & \mathbf{X}_{1}^{\perp}
\end{array}\right)\left(\begin{array}{cc}
\mathbf{U}_{1} & \mathbf{0} \\
\mathbf{0} & \mathbf{U}_{2}
\end{array}\right)\left(\begin{array}{l}
\mathbf{C} \\
\mathbf{S}
\end{array}\right) \mathbf{V}_{1}^{H} \mathbf{V}_{1} \mathbf{U}_{1}^{H}
\end{aligned}
$$

With (14), we have:

$$
\begin{array}{r}
\mathbf{X}(1)=\left(\begin{array}{ll}
\mathbf{X}_{1} \mathbf{X}_{1}^{\perp}
\end{array}\right)\left(\begin{array}{c}
\mathbf{X}_{1}^{H} \mathbf{X}_{2} \\
\left(\mathbf{X}_{1}^{\perp}\right)^{H} \mathbf{X}_{2}
\end{array}\right) \mathbf{V}_{1} \mathbf{U}_{1}^{H} \\
=\left(\begin{array}{ll}
\mathbf{X}_{1} \mathbf{X}_{1}^{\perp}
\end{array}\right)\left(\begin{array}{c}
\mathbf{X}_{1}^{H} \\
\left(\mathbf{X}_{1}^{\perp}\right)^{H}
\end{array}\right) \mathbf{X}_{2} \mathbf{V}_{1} \mathbf{U}_{1}^{H}=\mathbf{X}_{2} \mathbf{V}_{1} \mathbf{U}_{1}^{H}
\end{array}
$$

According to the definition of Grassmannia manifold, $\mathbf{X}_{2} \mathbf{V}_{1} \mathbf{U}_{1}^{H}$ is equivalent to $\mathbf{X}_{2}$. Therefore, Eq. (15) holds.

\section{Abbreviations}

UAV: unmanned aerial vehicle; MIMO: multi-input multi-output; 5G: 5th generation; CSI: channel state information; BER: bit-error-rate; MIMO-OFDM: MIMO orthogonal frequency division multiplexing; BS: base station; LOS: line of sight; NLOS: non-LOS; SNR: signal to noise ratio; SVD: singular value decomposition

\section{Acknowledgements}

Not applicable.

\section{Authors' contributions}

Dr. W. Zhou deduced most of the formulas and made most of the simulation experiments. Dr. X. Li verified the formulas and modeled the UAV-enabled MIMO system. Prof. H. Wu, Dr. Y. Xu, Dr. Q. Zhou, and Dr. Y. Rao analyzed the communication scenarios, arranged the data, analyzed computation complexity of the algorithm, and helped to revise this paper, respectively. All authors read and approved the final manuscript.

\section{Funding}

This work is supported by National Natural Science Foundation of China $(61601275,61471229)$, National Science Foundation of Guangdong Province (2019A1515011950, 2016A030313068), and Personnel Training Project of Guangzhou University (RP2020122).

\section{Availability of data and materials}

The authors state the data availability in this manuscript.

\section{Competing interests}

The authors declare that there is no conflict of interest regarding the publication of this paper.

\section{Author details}

${ }^{1}$ Department of Electronic Engineering, Nanjing Forestry University, Nanjing, China. ${ }^{2}$ Department of Electronic Engineering, Shantou University, Shantou, China. ${ }^{3}$ School of Electronic Engineering and Intelligence, Dongguan University of Technology, Dongguan, China. ${ }^{4}$ School of Computer Science and Cyber Engineering, GuangZhou University, GuangZhou, China.

Received: 21 September 2019 Accepted: 14 May 2020

Published online: 19 June 2020

\section{References}

1. X. Lai, Distributed secure switch-and-stay combining over correlated fading channels. IEEE Trans. Inf. Forensics Secur. 14(8), 2088-2101 (2019)

2. X. Lai, W. Zou, DF relaying networks with randomly distributed interferers. IEEE Access. 5, 18909-18917 (2017)

3. W. Wu, F. Zhou, P. Li, P. Deng, B. Wang, V. C. M. Leung, in 2019 IEEE International Conference on Communications (ICC), Energy-efficient secure NOMA-enabled mobile edge computing networks (IEEE, Shanghai, China, 2019), pp. 1-6

4. Z. Zhao, A novel framework of three-hierarchical offloading optimization for MEC in industrial loT networks. IEEE Trans. Ind. Inform. PP(99), 1-12 (2019)

5. J. Xia, Cache-aided mobile edge computing for b5g wireless communication networks. EURASIP J. Wirel. Commun. Netw. PP(99), 1-5 (2019) 
6. W. Wu, B. Wang, Y. Zeng, H. Zhang, Z. Yang, Z. Deng, Robust secure beamforming for wireless powered full-duplex systems with self-energy recycling. IEEE Trans. Veh. Technol. 66(11), 10055-10069 (2017)

7. W. Wu, F. Zhou, R. Q. Hu, B. Wang, Energy-efficient resource allocation for secure NOMA-enabled mobile edge computing networks. IEEE Trans. Commu. 68(1), 493-505 (2020)

8. B. Lu, Interference suppression by exploiting wireless cache in relaying networks. Phys. Commun. PP, 1-10 (2020)

9. S. Lai, Intelligent secure communication for cognitive networks with multiple primary transmit power. IEEE Access. PP(99), 1-7 (2020)

10. C. Komninakis, C. Fragouli, A. H. Sayed, R. D. Wesel, Multi-input multi-output fading channel tracking and equalization using Kalman estimation. IEEE Trans. Sig. Process. 50(5), 1065-1076 (2002). https://doi.org/10.1109/78.995063

11. J. Zhao, F. Gao, W. Jia, J. Zhao, W. Zhang, in 2017 IEEE International Conference on Communications (ICC), Channel tracking for massive MIMO systems with spatial-temporal basis expansion model (IEEE, Paris, 2017), pp. 1-5. https:// doi.org/10.1109/ICC.2017.7996914

12. X. Y. Yu, J. Wang, L. X. Yang, Time-varying channel tracking using sequential Monte Carlo filter in MIMO systems. J. Circ. Syst. 11(3), 18-21 (2006)

13. D. Zheng-cong, L. Li-xin, X. Li-jun, in 2009 International Conference on Communication Software and Networks, MIMO time-varying channel tracking with a polynomial channel model via particle filtering (IEEE, Chengdu, 2009), pp. 73-76. https://doi.org/10.1109/ICCSN.2009.26

14. J. Zhao, F. Gao, L. Kuang, Q. Wu, W. Jia, Channel tracking with flight control system for UAV mmwave mimo communications. IEEE Commun. Lett. 22(6), 1224-1227 (2018). https://doi.org/10.1109/LCOMM.2018.2824800

15. J. Zhao, W. Jia, Efficient channel tracking strategy for mmwave UAV communications. Electron. Lett. 54(21), 1218-1220 (2018). https://doi.org/10.1049/el.2018.6159

16. C. Tu, B. Champagne, in 2009 IEEE International Conference on Acoustics, Speech and Signal Processing, Subspace tracking of fast time-varying channels in precoded MIMO-OFDM systems (IEEE, Brighton, 2009), pp. 2565-2568. https://doi.org/10.1109/ICASSP.2009.4960146

17. J. Xia, Secure cache-aided multi-relay networks in the presence of multiple eavesdroppers. IEEE Trans. Commun. 67(11), 7672-7685 (2019)

18. J. Xia, When distributed switch-and-stay combining meets buffer in iot relaying networks. Phys. Commun. PP, 1-9 (2019)

19. X. Lin, Probabilistic caching placement in uav-assisted heterogeneous wireless networks. Phys. Commun. 33, 54-61 (2019)

20. L. Fan, N. Zhao, X. Lei, Q. Chen, N. Yang, G. K. Karagiannidis, Outage probability and optimal cache placement for multiple amplify-and-forward relay networks. IEEE Trans. Veh. Technol. 67(12), 12373-12378 (2018)

21. F. Shi, Secure probabilistic caching in random multi-user multi-uav relay networks. Phys. Commun. 32, 31-40 (2019)

22. X. Lin, MARL-based distributed cache placement for wireless networks. IEEE Access. 7, 62606-62615 (2019)

23. F. L. T. Li, C. Li, Manifold-based predictive precoding for the time-varying channel using differential geometry. Wirel. Netw. 22(8), 2773-2783 (2016)

24. W. Huang, Multi-antenna processing based relaying networks aided by wireless caching. Phys. Commun. PP, 1-10 (2020)

25. Y. Guo, Array signal processing assisted intelligent offloading strategy design in mobile edge computing networks for loT-based smart city. IEEE Access. PP(99), 1-7 (2020)

26. S. Schwarz, M. Rupp, Predictive quantization on the Stiefel manifold. IEEE Sig. Process. Lett. 22(2), 234-238 (2015) https://doi.org/10.1109/LSP.2014.2354258

27. K. He, Generic deep learning based linear detectors for MIMO systems over correlated noise environments. IEEE Access. PP(99), 1-9 (2020)

28. R. Zhao, Deep reinforcement learning based mobile edge computing for intelligent internet of things. IEEE Access. PP(99), 1-8 (2020)

29. F. Jiang, Y. Wang, C. Y. Yang, Performance analysis of downlink multi-user MIMO systems based on spatial-temporal correlated channels. J. Sig. Process. 23(4A), 390-393 (2007)

30. D. Tse, P. Viswanath, Fundamentals of Wireless Communications. (Cambridge University Press, Cambridge, 2004)

31. Y. Xu, Q-learning based physical-layer secure game against multi-agent attacks. IEEE Access. 7, 49212-49222 (2019)

32. C. Li, W. Zhou, Enhanced secure transmission against intelligent attacks. IEEE Access. 7, 53596-53602 (2019)

33. D. Deng, Link selection in buffer-aided cooperative networks for green loT. IEEE Access. PP(99), 1-8 (2020)

34. X. Wang, Joint resource allocation for cognitive OFDM-NOMA systems with energy harvesting in green loT. IEEE Access. PP(99), 1-8 (2020)

35. G. Liu, Deep learning based channel prediction for edge computing networks towards intelligent connected vehicles. IEEE Access. 7, 114487-114495 (2019)

36. J. Xia, Intelligent secure communication for internet of things with statistical channel state information of attacker. IEEE Access. 7(1), 144481-144488 (2019)

37. K. He, A MIMO detector with deep learning in the presence of correlated interference. IEEE Trans. Veh. Technol. PP(99), 1-5 (2019)

38. C. Li, Y. Xu, Protecting secure communication under UAV smart attack with imperfect channel estimation. IEEE Access. 6(1), 76395-76401 (2018)

39. Z. Zhao, Intelligent mobile edge computing with pricing in internet of things. IEEE Access. PP(99), 1-8 (2020)

40. C. Li, Cache-enabled physical-layer secure game against smart uav-assisted attacks in b5g noma networks. EURASIP Journal on Wireless Communications and Networking. PP(99), 1-5 (2019)

41. J. Yang, D. B. Williams, Transmission subspace tracking for MIMO systems with low-rate feedback. IEEE Trans. Commun. 55(8), 1629-1639 (2007). https://doi.org/10.1109/TCOMM.2007.902598

42. D. Zhu, Y. Zhang, G. Wang, M. Lei, Grassmannian subspace prediction for precoded spatial multiplexing MIMO with delayed feedback. IEEE Sig.Process. Lett. 18(10), 555-558 (2011). https://doi.org/10.1109/LSP.2011.2162947

43. H. Hu, Sparse discriminative multimanifold Grassmannian analysis for face recognition with image sets. IEEE Trans Circ. Syst. Video Technol. 25(10), 1599-1611 (2015). https://doi.org/10.1109/TCSVT.2014.2367357 
44. T. Connie, M. K. O. Goh, A. B. J. Teoh, A Grassmannian approach to address view change problem in gait recognition. IEEE Trans. Cybernet. 47(6), 1395-1408 (2017). https://doi.org/10.1109/TCYB.2016.2545693

45. A. Edelman, T. A. Arias, S. T. Smith, The geometry of algorithms with orthogonality constraints. SIAM J. Matrix Anal. Appl. 20(2), 303-353 (1998)

46. R. A. Horn, C. R. Johnson, Matrix Analysis. (Cambridge University Press, Cambridge, 2012)

47. G. H. Golub, C. F. Van Loan, Matrix Computations, 4th Edition. (The Johns Hopkins University Press, Baltimore, 2013)

48. K. Lee, Y. Lee, Effect of antenna correlation on capacity of opportunistic eigenbeamforming in Rayleigh fading channels. Electron. Lett. 46(20), 1365-1366 (2010). https://doi.org/10.1049/el.2010.2195

\section{Publisher's Note}

Springer Nature remains neutral with regard to jurisdictional claims in published maps and institutional affiliations.

\section{Submit your manuscript to a SpringerOpen ${ }^{\circ}$} journal and benefit from:

- Convenient online submission

Rigorous peer review

Open access: articles freely available online

- High visibility within the field

- Retaining the copyright to your article

Submit your next manuscript at $\gg$ springeropen.com 